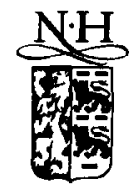

ELSEVIER

\title{
Radiation hardness of undoped BGO crystals
}

\author{
S.K. Sahu ${ }^{\mathrm{a}, \mathrm{d}, *}$, K.C. Peng ${ }^{\mathrm{a}}$, H.C. Huang ${ }^{\mathrm{a}}$, C.H. Wang ${ }^{\mathrm{c}}$, Y.H. Chang ${ }^{\mathrm{b}}$, W.S. Hou ${ }^{\mathrm{a}}$, K. Ueno ${ }^{\mathrm{a}}$, \\ F.I. Chou ${ }^{\mathrm{e}}, \mathrm{Y} . \mathrm{Y}$. Wei ${ }^{\mathrm{e}}$ \\ ${ }^{2}$ National Taiwan University, Taipei, Taiwan. China \\ ${ }^{\mathrm{b}}$ National Central Uninersity, Chung-Li, Taiwan, China \\ ${ }^{'}$ National Lien Ho College of Technology and Commerce, Miao Li, Taiwan, China \\ dNational Laboratory for High Energy Physics. KEK, Tsukuba, Ibaraki, Japan \\ 'Nuclear Science and Technology Development Center, NTHU, Hsinchu, Taiwan, China
}

Received 20 December 1996

\begin{abstract}
We measured the radiation hardness of undoped BGO crystals from two different manufacturers. Such crystals are proposed to be used in a small-angle calorimeter of the BELLE detector of the KEK B-factory. Transparency and scintillation light output of the crystals were monitored to see the effect of radiation damage. The crystals show considerable radiation hardness up to $10.2 \mathrm{Mrad}$ equivalent dose, which is much higher than the maximum expected dosage of $500 \mathrm{krad}$ per year of running at BELLE.
\end{abstract}

Keywords: Radiation hardness; Radiation damage; BGO; Undoped; Calorimeter

\section{Introduction}

Bismuth Germanate $\left(\mathrm{Bi}_{4} \mathrm{Ge}_{3} \mathrm{O}_{12}\right)$ crystals, commonly known as BGO, have been extensively used for electromagnetic calorimetry in high-energy physics experiments [1]. Advantages of $\mathrm{BGO}$ are its excellent $e / \gamma$ energy resolution $(0.3-1 \% / \sqrt{(E}(\mathrm{GeV}))$, high-density, short radiation length, large refractive index, suitable scintillating properties (fast decay time of about $300 \mathrm{~ns}$ and peak scintillation at about $450 \mathrm{~nm}$ ) and non-hygroscopic nature. It is therefore the best candidate for EM calorimetry in collider experiments, especially where space imposes a serious constraint. Such a calorimeter has been proposed, for example, for the BELLE detector at KEK $B$-factory to cover very small angles around the beam pipe [2]. Radiation level at such small angles is rather high in B-factories due to intense flux of photons and electrons generated by Bhabha events and spent-electron background events [3].

Impure (doped) BGO crystals are known to get damaged by radiation [4], even at $1 \mathrm{krad}$, while pure

\footnotetext{
* Correspondence address: Department of Physics and Astronomy, 2505 Correa Road, University of Hawaii, Honolulu, HI 96822, USA; E-mail: sahu@uhhepi.phys.hawaii.edu.
}

ones from certain manufacturers have shown considerable hardness even at $100 \mathrm{Mrad}$ of irradiation [5]. A detailed study of the effect of different impurities on radiation hardness of BGO has been done by $Z$ hu et al. [4]. Doped BGO crystals have about the same decay time and emission peaks as pure ones, but the amount of scintillation can be very different depending on the species and quantity of doping. Pure BGOs require about $300 \mathrm{eV}$ per scintillation, whereas doped ones may need anywhere between 60 and $200 \mathrm{eV}$ per scintillation [6].

We, therefore, being proponents of the above-mentioned calorimeter at KEK B-factory, set forth to determine the radiation hardness of some pure BGO samples of dimensions comparable to our proposed detector.

The crystals were irradiated up to a $10.2 \mathrm{Mrad}$ equivalent dose, ${ }^{1}$ and the two properties, scintillation light output and transparency, were monitored to measure the effect of the radiation damage.

The experimental setup and results of our experiment are described in the following sections.

\footnotetext{
${ }^{1}$ We define the equivalent dose as the amount of dose that would have been absorbed by water if placed at the same position as the sample. Absolute dose absorbed by the BGO crystal is estimated to be about 1.3 times the equivalent dose.
} 


\section{Experimental setup}

Undoped (pure) BGO crystals of sizes $12 \times 1 \times 1 \mathrm{~cm}^{3}$ and $2.5 \times 1 \times 1 \mathrm{~cm}^{3}$ were obtained from the Institute of Single Crystals, Kharkov, Ukraine; and of sizes $10 \times 1 \times 1 \mathrm{~cm}^{3}$ and $2.5 \times 1 \times 1 \mathrm{~cm}^{3}$ from the Institute of Inorganic Chemistry, Novosibirsk, Russia, for this irradiation test.

Scintillation light output and transparency were the two properties of the BGO samples that were monitored as functions of radiation dose. Schematic diagram of the setup for these measurements is given in Fig. 1. A wooden guide and a photo-multiplier tube (PMT, Hamamatsu R329-05, 2 in. diameter) were fixed on an aluminum base. The crystal sample was placed on the guide for each measurement, and would touch the PMT window. The guide was used in order to make sure that the crystal always touched exactly the same place of the PMT window. The aluminum base along with this setup was placed in a light-tight box, and was slightly slanted so that the crystal would lean on the PMT by gravity. This way a uniform spacing between the crystal surface and PMT window could be guaranteed.

Blue LEDs $(\lambda \pm \Delta \hat{\lambda}=440 \pm 15 \mathrm{~nm})$ were fixed at three positions, $A, B$ and $C$. They could be pulsed by a pulser (HP 8112A) outside of the setup. Each pulse would give a burst of photons into the crystal, and some of them would reach the PMT. The crystals were not wrapped with any kind of reflector and optimum care was taken not to leave any finger print or dust on the crystal surface. This arrangement would then guarantee that any deterioration in the number of photo-electrons collected by the PMT would be a consequence of the change in transparency of the crystal. The pulser to light I.E.Ds was so adjusted that about 100 photo-electrons were collected by the PMT prior to any irradiation. We define the Relative transparency of a crystal at a certain point ( $A$, $B$ or $C$ ) as the ratio of the average number of photoelectrons collected by the PMT through the irradiated sample to that through the reference sample.

Small slots were prepared in the wooden guide, at positions $X, Y$ and $Z$, where a ${ }^{60} \mathrm{Co}$ source could be installed on top of the crystal. The scintillation light produced in the bulk of the crystal by the $\gamma$-rays from this source were collected by the PMT. Any reduction in the number of photo-electrons would reflect the effect of radiation damage. Of course, for the crystals shorter than shown in Fig. 1, data for position $Z$ or/and $Y$ were not available. We define the Relative light output of a crystal at a certain point $(X, Y$ or $Z)$ as the ratio of the average number of photo-electrons collected by the PMT through the irradiated sample to that through the reference sample.

The signal from the PMT was digitized with a LeCroy CAMAC ADC 2249A. The signals generated by the LED bursts were gated by the trigger from the pulser, whereas the scintillation signals produced with ${ }^{60} \mathrm{Co}$ were gated by self-trigger. The CAMAC crate was controlled by a PC-based data acquisition system.

The number of photo-electrons was estimated from the average charge produced by the PMT and digitized by the ADC.

Normalizing the data with a reference crystal, as above, cancels out the systematic errors due to change in PMT gain, temperature, humidity, etc. The reference crystal was never irradiated, but was kept under

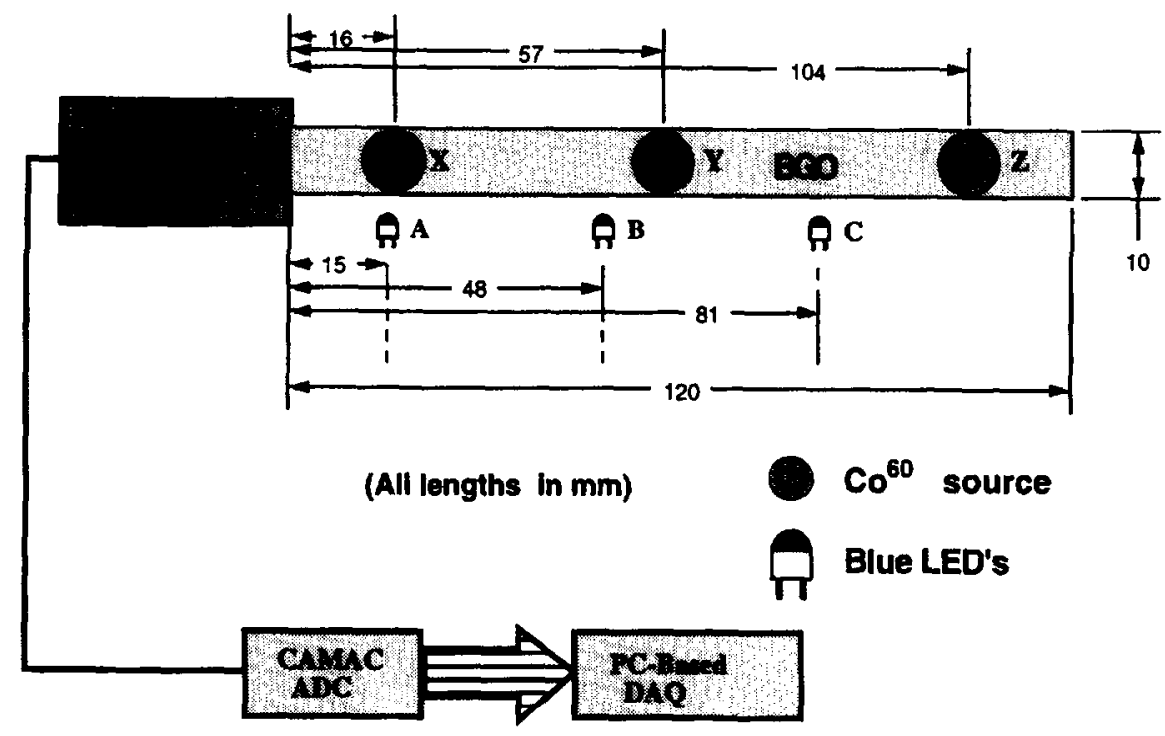

Fig. 1. Schematic diagram of the light output and transparency measurement system for the BGO crystals. 
the same environmental conditions as the irradiated crystals.

The $\gamma$-ray irradiation was conducted at the irradiation facility of National Tsing Hua University, Hsin-Chu, Taiwan. The source was ${ }^{60} \mathrm{Co}$ with an activity of 1320 Curie. The length of the crystals was oriented perpendicular to the source, thus simulating the real situation in our proposed detector, where crystals would tower towards the interaction point. Referring to Fig. 1, during irradiation, the sides of the crystals facing the PMT were kept farthest from the ${ }^{60}$ Co source. Hence, the side close to the PMT received much less dose than the other side. This issue is addressed in Section 3 and in Fig. 4.

The distance of the crystal from the radiation source was varied such that each step of dose would be obtained in about $1 \mathrm{~h}$. Maximum rate of dose imposed in this experiment was about $1 \mathrm{Mrad} / \mathrm{h}$, when the crystal was very close to the source.

Immediately after each irradiation, the samples were taken out from the irradiation cell to a near-by experi- mental booth where scintillation and transparency measurements were carried out. These measurements would typically take about $1 \mathrm{~h}$, and then the samples could be put in the cell for further irradiation.

\section{Result}

The crystals were irradiated in 8 steps upto $10.2 \mathrm{Mrad}$. After each step the scintillation light output and transparency were checked, as described in the previous section.

Scintillation relative light output for source positions $X$ and $Y$ for long crystals from Novosibirsk and Kharkov are shown in Fig. 2(a) and (b), respectively. Light output for the source position $Z$ is shown in Fig. 2(c) (for Kharkov crystal only). For short crystals (position $X$ only) the light output is given in Fig. 2(d).

Relative transparency measured by LED pulsing for positions $A, B$ and $C$ are shown in Figs. 3(a)-(c), respectively, for longer crystals from Novosibirsk and Khar-

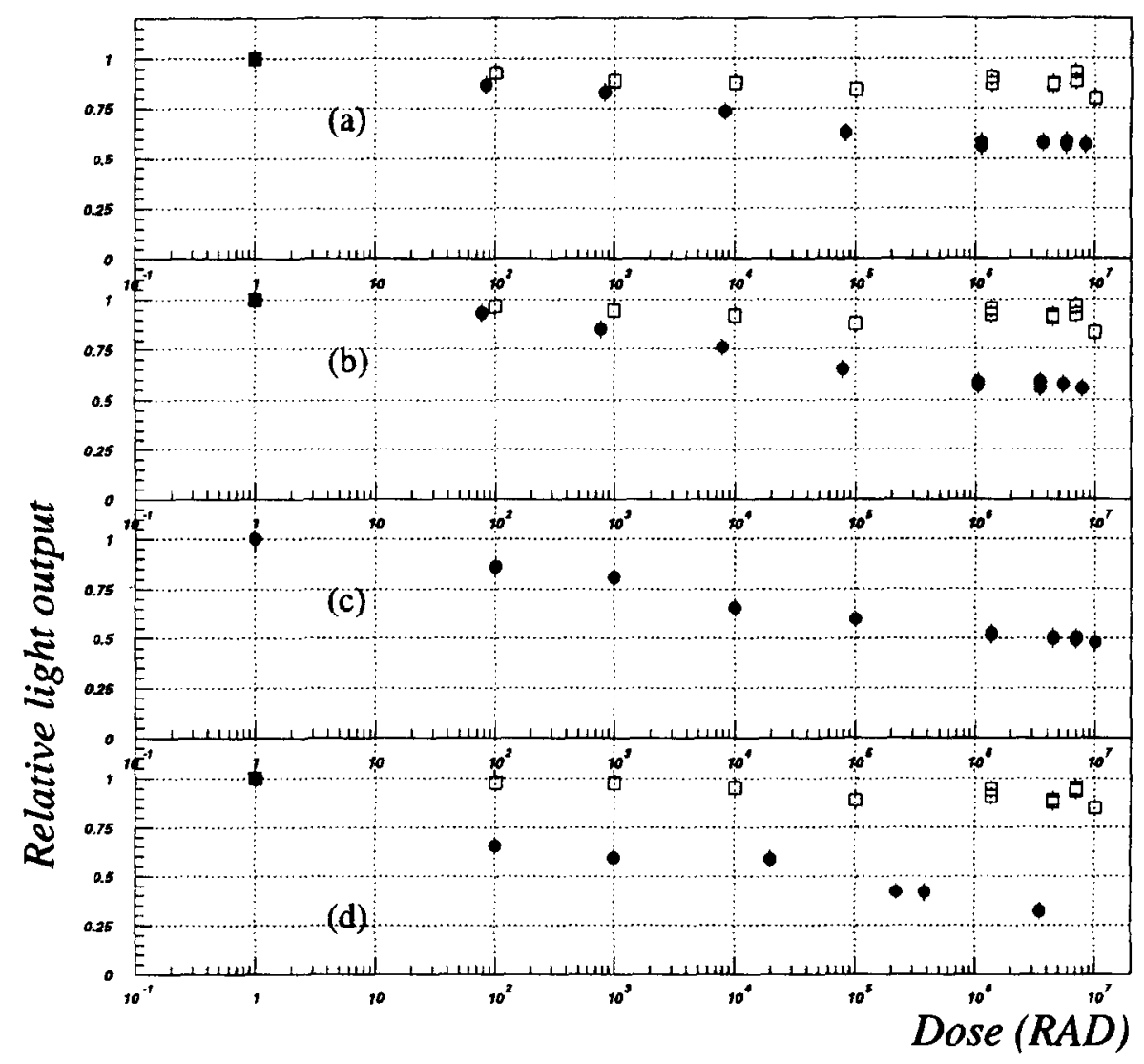

Fig. 2. Relative light output produced by the $\mathrm{BGO}$ crystals when scintillated with gamma rays from ${ }^{60} \mathrm{Co}$ source for positions (a) $X$, (b) $Y$, (c) $Z$ as a function of irradiation dose, (d) short crystals. Open squares are for Novosibirsk and closed circles are for Kharkov Crystals. 


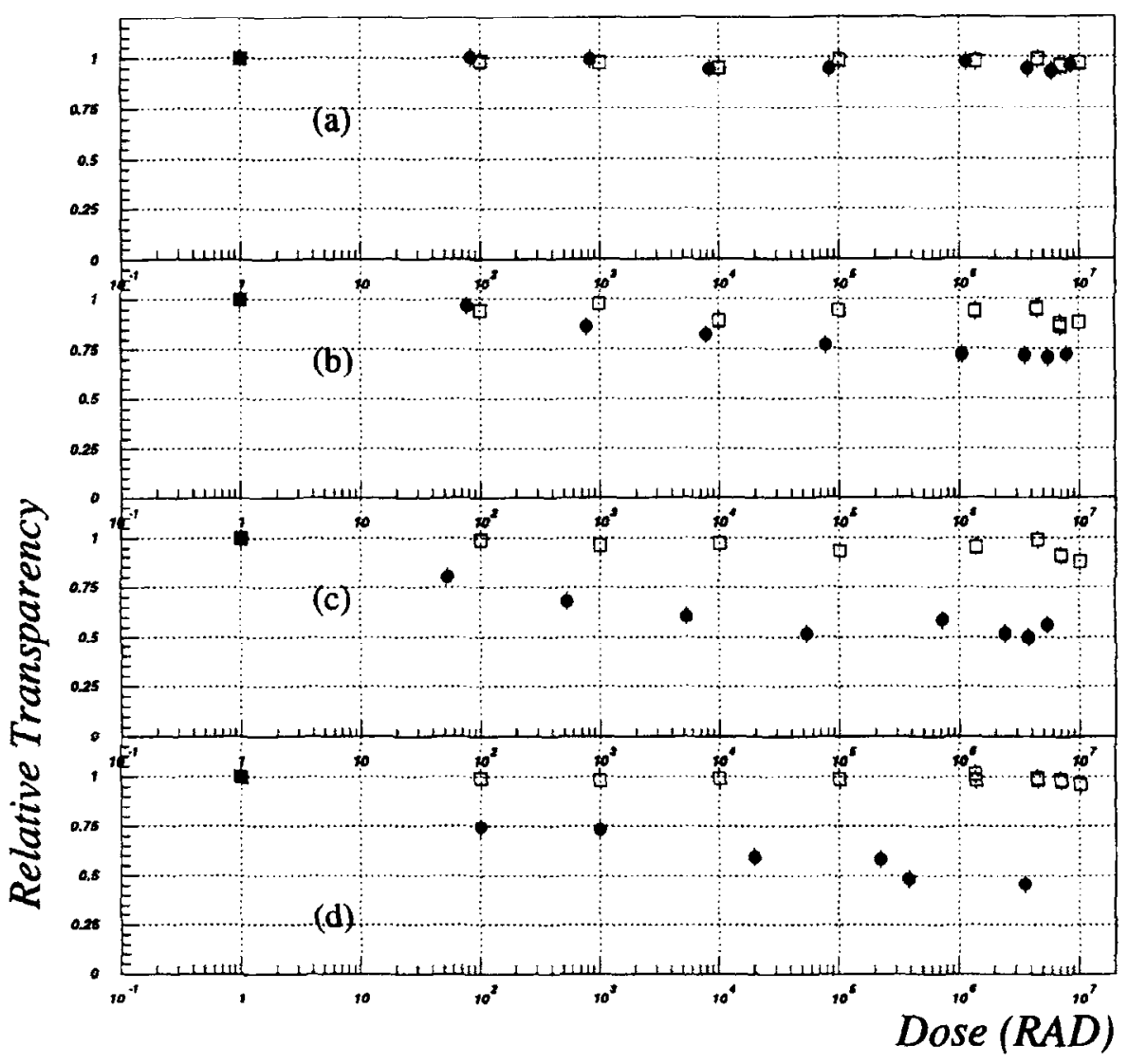

Fig. 3. Relative transparency of BGO crystals, when lit with blue LEDs for positions (a) $A$, (b) $B$, (c) $C$ as a function of irradiation dose, (d) short crystals. Open squares are for Novosibirsk and closed circles are for Kharkov Crystals.

kov. The same quantity for the short crystals from both manufacturers is plotted in Fig. 3(d).

Values plotted in these figures are the dose received at the face of the crystal, i.e., at the end closest to the source. Amount of the dose received at other parts of the crystal can be calculated from Fig. 4.

The first point in these figures actually corresponds to the values prior to any irradiation : 0 rad. To avoid the trouble of plotting a zero in log-scale, we have plotted it at $1 \mathrm{rad}$ value. Values for both crystals are normalized to unity for better comparison of effects due to radiation damage.

There is a systematic error of about $2 \%(5 \%)$ for doses below $1 \mathrm{Mrad}$ (above $1 \mathrm{Mrad}$ ) in estimation of the dose (abscissa in Figs. 2 and 3). Statistical error in measuring the relative light output and relative transparency is $3 \%$. Total systematic error caused by the placement of samples in the measuring device, and due to the post-irradiation fluoroscence was estimated to be less than $3 \%$.

Double entries at same irradiation dose for some points means that the crystal was kept unirradiated for about $60 \mathrm{~h}$ (because of the week-end shut down of the irradiation facility) and the two points give the measured quantity just before and after this idling period. The second point is always higher than the first point, which may be because of the fact that the crystals recover from the radiation damage to some extent during this time interval.

Since the length of the crystal was perpendicular to the ${ }^{60} \mathrm{Co}$ source, the dose absorbed was not uniform along the length of the crystal. Furthermore, the source itself was not a point, but had a cylindrical shape. A GEANT [7] simulation was performed, taking these two facts into account, in order to determine the variation of the absorbed dose along the length of the crystal. The result is plotted in Fig. 4. Fraction of the total absorbed dose (with ${ }^{60} \mathrm{Co}$ irradiation) in every $0.5 \mathrm{~cm}$ interval of length is plotted against the length of a $10 \mathrm{~cm}$ long BGO crystal. The first bin is normalized to unity, which corresponds to the value of the dose plotted in Figs. 2 and 3. The absorbed dose along the length of the crystal shows a nearexponential dependence, as expected for such low-energy $(1.173$ and $1.333 \mathrm{MeV}) \gamma$-rays.

The lengths of the samples from Novosibirsk and Kharkov were a little different, and the entries for the 


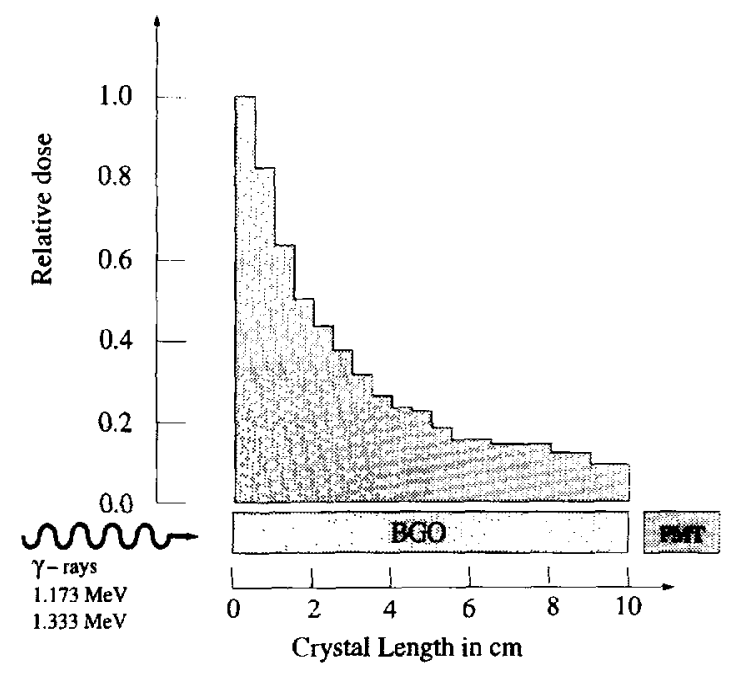

Fig. 4. Variation of absorbed dose along the length of a $\mathrm{BGO}$ crystal, when irradiated with $\gamma$-rays from ${ }^{60} \mathrm{Co}$. The estimation is obtained with a Geant simulation, taking finite source size into account. First bin of the histogram is normalized to unity, since the equivalent dose is measured at this point.

dose in Figs. 2 and 3 were corrected by weights taken from Fig. 4.

It is seen clearly from Figs. 2 and 3 that pure BGO crystals from Novosibirsk are quite radiation hard at least upto $10.2 \mathrm{Mrad}$ equivalent dose (with a maximum of $15 \%$ damaging effect), while Kharkov crystals degrade by more than $40 \%$ in terms of scintillation light output and transparency at such a dose.

\section{Conclusion}

We conclude that the pure BGO crystals from Novosibirsk are radiation hard upto $10.2 \mathrm{Mrad}$ of equivalent dose, hence, can be used in the high-irradiation environments, such as B-factories.

\section{Acknowledgements}

This work was initiated by Prof. J.C. Peng of LANL. We are grateful to him for giving valuable comments on the manuscript. We would like to thank the CsI Calorimeter subgroup of the BELLE Collaboration of KEK B-Factory for its help in this project. Prof. M. Fukushima of KEK and Prof. A. Bondar of Budker Institute have especially been very helpful and given many useful advices. We are grateful to the staff-members Dr. F.I. Chou, Mr. M.T. Duo, Mr. K.W. Fang and Mr. Y.Y. Wei of the irradiation facility (NSTDC) at National Tsing Hua University, Hsin-Chu, Taiwan for their help and co-operation. This experiment was partially supported by the Grant NSC 85-2112-M-002-034 of the Republic of China.

\section{References}

[1] L3 Collaboration, R. Sumner et al., Nucl. Instr. and Meth. A 265 (1988) 252 .

H. Hayashii et al., Nucl. Instr. and Meth. A 316 (1992) 202; D.N. Grigoriev et al., IEEE Trans. Nucl. Sci. NS-42 (1995) 505.

[2] BELLE Progress Report, KEK Progress Report 96-1 (1996): Conceptual Design of the Extreme Forward Calorimeter for BELLE, W.S. Hou et al., BELLE Note 90 (1995).

[3] T.E. Browder (Ed.), Proc. Workshop on Backgrounds at the Machine Detector Interface, UH-511-838-96.

[4] R.Y. Zhu et al., Nucl. Instr. and Meth. A 302 (1991) 69; P. Lecoq, P.J. Li and B. Rostaing, ibid A 300 (1991) 240; Z.Y. Wei et al., ibid A 297 (1990) 163;

Z.W. Yin et al., ibid A 275 (1989) 273;

C. Bieler et al., ibid A 234 (1985) 435;

G.J. Bobbink et al., ibid A 227 (1984) 470;

M. Kobayashi et al., ibid A 206 (1983) 107;

A.J. Caffrey et al., IEEE Trans. Nucl. Sci. NS-33 (1986) 230.

[5] V.V. Yanovskii, V.A. Chizhov, V.M. Skorikov, Nucl. Instr. and Meth. A 309 (1991) 596.

[6] E. Lorenz, Nucl. Instr. and Meth. A 225 (1984) 500.

[7] GEANT Detector Description and Simulation Tool, Application Software Group, CERN; CERN Program Library Long Writeup W5013. 